\title{
ASPETTI DECORATIVI AGLI INIZI DELLA FABBRICA DEL DUOMO
}

\author{
MARCO ROSSI (*)
}

RIASSUNTO. - Le prime fasi della costruzione del Duomo di Milano rivelano nell'ambito del tardogotico europeo una significativa integrazione fra architettura e decorazione rispetto a una prevalente concezione della scienza architettonica. Questa tensione verso l'unità delle arti è particolarmente promossa da Giovannino de Grassi, attraverso i procedimenti ideativi e creativi da lui adottati e soprattutto i rapporti tra la sua intensa attività progettuale e le infinite possibilità offerte dai suoi disegni. Le invenzioni di Giovannino, relative a guglie e capitelli, sculture e vetrate, dipinti e oreficerie, sviluppano le infinite possibilità offerte dal disegno tardogotico e l'illimitata applicabilità delle soluzioni figurative create, dall'architettura alla decorazione, dalla realtà all'immaginazione.

$* * *$

ABSTRACT. - The first stages of the construction of the Cathedral of Milan in the Late Gothic reveal a significant European integration between architecture and decoration rather than a prevailing conception of architectural science. This tension for the unity of the arts is particularly promoted by Giovannino de Grassi, through the ideational and creative processes of the master, above all the relations between his intense designing activity in the field of architecture and the infinite possibilities offered by its drawings. The Giovannino's inventions produce spires and capitals, sculptures and stained glass, paintings and goldsmithings. The infinite possibilities offered by Late Gothic drawing and the unlimited applicability of its figurative solutions develop a continuous symbiosis between architecture and decoration, reality and imagination.

Molto spesso la letteratura critica relativa al Duomo di Milano si è soffermata su presunte anomalie del monumento rispetto a una generica e per certi versi astratta concezione delle cattedrali gotiche, evitando in tal senso lo sforzo di storicizzare la fabbrica nel contesto di un

(*) Università Cattolica del Sacro Cuore; Accademia Ambrosiana di Studi Borromaici di Milano. 
particolare momento del tardogotico europeo e di qualificarne le peculiarità nell'ambiente milanese.

L'attenta disamina delle vicende costruttive è in parte ancora da mettere in luce, correlando fonti documentarie e analisi degli interventi architettonici e decorativi, oltre che del contesto storico-artistico locale e internazionale. Allo stato degli studi risultano comunque evidenti numerosi tentativi del cantiere, che poteva essere condotto senza problemi secondo la tradizionale esperienza delle maestranze campionesi, di aggiornamenti in prospettiva europea, nella consapevolezza di voler partecipare al dibattito artistico internazionale. Certamente le ambizioni politiche viscontee, di Gian Galeazzo in primis, ${ }^{1}$ richiedevano e favorivano tali intenzioni, ma non bisogna pensare che ne esaurissero le potenzialità culturali e gli orizzonti innovativi, a partire dalla circolazione di maestranze e modelli.

Questo implicava innanzi tutto far propria la concezione della cattedrale gotica non genericamente intesa, ma aggiornata ai cantieri europei attivi a fine Trecento, e non solo quale occasione di prestigio politico e culturale, come interessava soprattutto a Gian Galeazzo, ma di ripensamento di una tradizione religiosa e civile, che aveva visto la città e l'episcopato di Milano tra i più alti protagonisti del Medioevo e la cultura artistica lombarda connotata da significative peculiarità costruttive, organizzative e formali, apprezzate e diffuse in molti ambienti.

Il complesso episcopale di Milano aveva infatti rivestito un ruolo fondamentale fin dall'età ambrosiana e le cattedrali di S. Tecla e di S. Maria Maggiore custodivano un immenso tesoro di opere, di tradizione e di consapevolezza storica: non poteva dunque essere affrontata a cuor leggero una così complessa operazione di rinnovamento, che coinvolgeva la coscienza, le aspettative e gli interessi di un insieme estremamente articolato di realtà civili e religiose, come riveleranno le vicende della fabbrica. E al tempo stesso le istanze di rinnovamento non potevano essere imposte a un mondo così vario e strutturato - basti pensare agli ordini dei canonici che facevano capo alle cattedrali ${ }^{2}-$ ma occor-

1 Cfr. E.S. Welch, Art and Authority in Renaissance Milan, New Haven-London 1995; F. Tasso. Il progetto 'della memoria'. Testimonianze documentarie e presenze sul territorio per una ricostruzione dell'attività di committente di Gian Galeazzo Visconti, in Nuova Rivista storica, LXXXVI, 2002, 1, pp. 129-154.

2 E. Cattaneo, Il clero e la cura pastorale nell' antico Duomo S. Tecla, Milano 1950; Id., Istituzioni ecclesiastiche milanesi, in Storia di Milano, IV, Milano 1954, pp. 651-656; 
reva che la consapevolezza della propria tradizione storica accogliesse le istanze provenienti dai grandi centri del gotico europeo: ad esempio le sollecitazioni di un pensiero teologico maturato nelle grandi scuole episcopali d'oltralpe, intese a percepire la cattedrale come microcosmo che svela l'ordine naturale dell'universo, in una continua apertura della ragione all'infinito, sollecitata dalla natura e dall'arte (invisibilia per visibilia); laddove invece a Milano l'idea di microcosmo era piuttosto percepita nella declinazione di un'ordinata amministrazione canonica e liturgica e nei rapporti tra potere civile e religioso, espressioni terrene di una gerarchia celeste. ${ }^{3}$ Questo non impedì l'adesione al linguaggio gotico internazionale, anzi intensificò il dibattito tecnico e critico, giungendo in alcuni casi a vertici considerevoli, come nel caso del recupero di fonti platoniche nella sanzione geometrica dell'alzato della cattedrale operata dallo Stornaloco, ${ }^{4} \mathrm{o}$ ancor più nella raffinata indagine formale della natura, nell'unità delle arti e nella tensione alla perfezione espressa dal disegno, promosse da Giovannino de Grassi nel cantiere del Duomo. 5

Probabilmente ci fu un cambio d'indirizzo culturale ed artistico dopo l'avvio di una nuova cattedrale ancora in cotto, secondo la tradizione gotica lombarda, come sembrano svelare i resti ritrovati qualche decennio fa nella sacrestia settentrionale ${ }^{6}$ ma si tratta di aspetti sui quali occorrerà approfondire le ricerche. Per ora osserviamo che il nuovo cantiere internazionale, che si qualifica per l'utilizzo del marmo di Candoglia e la presenza di maestranze straniere, opera fin da subito con in-

A. Ambrosioni, Milano e i suoi vescovi, in Atti dell'XI Congresso Internazionale di Studi sull'Alto Medioevo (Milano 1987), Spoleto 1989, pp. 291-326; M. Rossi. Le cattedrali perdute: il caso di Milano, in Medioevo: l'Europa delle cattedrali, Atti del IX Convegno Internazionale di Studi (Parma 2006), Milano 2007, pp. 288-236.

3 Cfr. A. Pracchi, La cattedrale antica di Milano. Il problema delle chiese doppie fra tarda antichità e medioevo, Bari 1996.

4 E. Battisti, Avanguardia e conservatorismo nella storia del Duomo di Milano, in Il Duomo di Milano, Atti del Congresso internazionale a cura di M.L. Gatti Perer (Milano 1968), Milano 1969, p. 47; M. Rossi. La correlazione di problemi statici, proporzionali e simbolici nelle prime proposte per la costruzione del tiburio del Duomo, in Arte Lombarda, 58/59, 1981, pp. 24-25.

5 M. Rossi, Giovannino de Grassi. La corte e la cattedrale, Cinisello Balsamo 1995; Id., Il cantiere del Duomo di Milano e l'unità delle arti, in Lombardia gotica e tardogotica. Arte e architettura, a cura di M. Rossi, Milano 2005, pp. 219-235.

6 C. Ferrari da Passano, Le origini lombarde del Duomo, Milano 1973. 
teressanti scambi di modelli e intrecci di competenze: infatti il 9 febbraio 1387 viene pagato un orafo tedesco, tale Anechino, qui fecit tiborium unum pombli.

Il processo d'internazionalizzazione del cantiere risulta fin da subito intenso, anche se sfuggono i tramiti dei primi contatti, probabilmente favoriti dalla politica matrimoniale e dalla diplomazia viscontea. L'orientamento prevalente verso i cantieri mitteleuropei piuttosto che verso il mondo francese, al quale Gian Galeazzo era particolarmente legato, sembrerebbe indotto dal suo obiettivo politico principale, quello cioè di ottenere il titolo ducale dall'imperatore.

Occorre osservare che accanto a vivaci contrasti tra maestranze locali e straniere, spesso favoriti dalla stessa Fabbrica per ottenere pareri tecnici sui lavori in corso, si assiste a interessanti esperienze di integrazione, che sembrano anche in grado di generare peculiari soluzioni formali, difficilmente rintracciabili in altri cantieri. Ad esempio lo scultore Hans Fernach, che è documentato la prima volta in Duomo il 12 luglio 1387 insieme ai lapicidi campionesi Albertolo, Maxa, Lazzaro e Marchese ${ }^{8}$ risulta coadiuvato nella realizzazione del suo capolavoro, il portale della sacrestia meridionale (Fig. 1 e Fig. 2), da molti lapicidi locali dipendenti dalla Fabbrica: Antonio da Campione, Guido de Branchino, Pietro Grexino e Antonio Giulini, dal fabbro Antonio da Paderno e dai carpentieri Domenico da Novate e Giacomo da Carona. ${ }^{9}$

La sovrapporta del Fernach è caratterizzata da un ricco sviluppo iconografico e decorativo, in origine previsto ancor più articolato, se non fossero intervenuti Giacomo da Campione e Giovannino de Grassi in abreviando opus designamenti. ${ }^{10}$ L'intensa densità plastica del complesso oscilla fra l'aspetto stereometrico di alcune figure e l'addolcimento di lineamenti e panneggi, con una chiara impronta dello Schöne Stil parleriano, favorendo alcuni arcaismi campionesi nel gusto per la decisa strutturazione dei personaggi, ma introducendo al tempo stesso nuove morbidezze formali insieme al più noto gusto francesizzante per profili taglienti e affilati.

7 Annali della Fabbrica del Duomo di Milano dall'origine fino al presente pubblicati a cura della sua amministrazione, Appendici, I, Milano 1883, p. 14. Cfr. E.S. Welch, Art and Authority, cit., p. 88.

8 Annali, App., I, p. 22.

9 Milano, Archivio della Fabbrica del Duomo, Registro 16. Cfr. M. Rossi. Architettura e scultura tardogotica tra Milano e l'Europa. Il cantiere del Duomo alla fine del Trecento, in Arte Lombarda, 126, 1999, p. 5.

10 Annali, I, p. 101. 


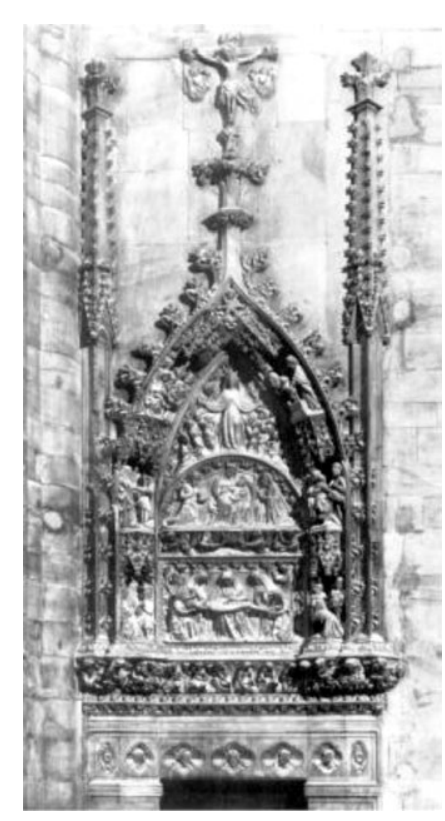

Fig. 1. Milano, Duomo. Hans Fernach, sovrapporta della sacrestia meridionale.

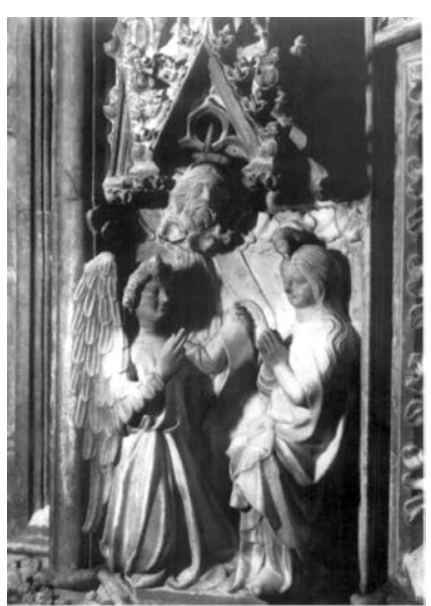

Fig. 2. Milano, Duomo. Hans Fernach, sovrapporta della sacrestia meridionale particolare dell'Annunciazione.

Il confronto con il portale della sacrestia settentrionale di Giacomo da Campione (Fig. 3) mette in evidenza la diversa impaginazione compositiva e le varianti formali, svelando, a proposito del Fernach, indubbi modelli mitteleuropei, laddove Giacomo rimaneva fedele a una più generica e diffusa impronta oltralpina di gotico slanciato e svettante. La rigogliosa densità plastica e figurativa della sovrapporta meridionale introduce nuove indicazioni e riferimenti parleriani, come può dimostrare il confronto con i portali esterni del coro delle cattedrali di Friburgo e di Ulm, con le quali erano intensi i rapporti del cantiere milanese. ${ }^{11}$

L'intreccio d'influssi e riferimenti stilistici e culturali, pur sempre nell'ambito di un generale gusto tardogotico europeo, che produce però sfumature diverse legate anche alle tradizioni locali dei vari terri-

11 Cfr. M. Rossi, Architettura e scultura tardogotica tra Milano e l'Europa. Il cantiere del Duomo alla fine del Trecento, in Arte Lombarda, 126, 1999, 2, pp. 5-29. 
tori, emerge ancor più chiaramente nei numerosissimi peducci che decorano l'alto basamento del Duomo (Fig. 4) e poi nelle mensole del primo ordine di statue (Fig. 10). Riguardo ai primi sembra aver avuto una certa importanza la presenza a Milano nel 1391 di Lasse d'Ungheria, ${ }^{12}$ che probabilmente prende il posto proprio del Fernach quando questi viene inviato in Germania per chiamare un ingegnere, quasi certamente Ulrich von Einsingen. Alcuni confronti con gli importanti frammenti scultorei ritrovati nel 1974 durante lavori nel primo cortile del palazzo reale di Buda, ora conservati nel Történeti Múzeum, permettono d'individuare a Milano alcune tipologie formali e stilistiche simili.

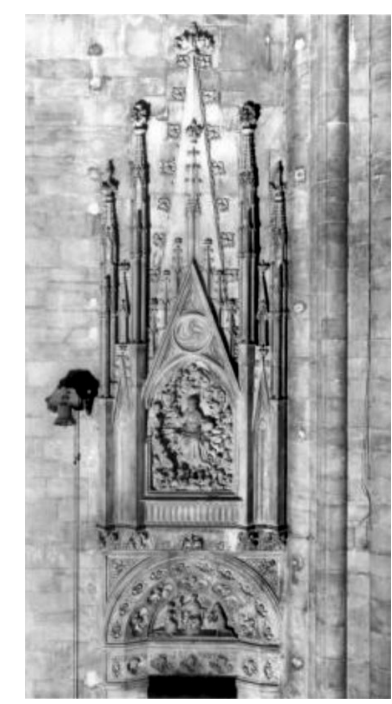

Fig. 3. Milano, Duomo. Giacomo da Campione, sovrapporta della sacrestia settentrionale.

Vale la pena ribadire che tale complesso di varianti decorative emerge fin dagli inizi della fabbrica del Duomo e risulta strettamente correlato alla qualità architettonica della fabbrica, non come qualcosa di aggiunto in fase d'opera, ma inerente il progetto stesso e in molti casi

12 M.G. Agghàzy, Lasse d'Ungheria e i rapporti tra Milano e Buda nel 1391, in Il Duomo di Milano, Atti del Congresso internazionale a cura di M.L. Gatti Perer, Milano 1969, I, pp. 89-94; M. Rossi. Maestranze ungheresi nel Duomo di Milano, in Arte Lombarda, 139, 2003, 3, pp. 28-34. 
direttamente le maestranze, non solo la committenza: di nuovo una conferma di quella "unità delle arti" all'interno del cantiere che vince ogni astratta concezione di scienza architettonica a favore di una feconda integrazione tra architettura e decorazione. L'autorevolezza dei protagonisti non è relegata a uno specifico ambito specialistico, ma alla loro capacità di affrontare problemi diversi, come dimostra ad esempio la partecipazione del noto scultore Bonino da Campione al dibattito del 20 marzo 1388 su eventuali errori d'impostazione della fabbrica. ${ }^{13}$

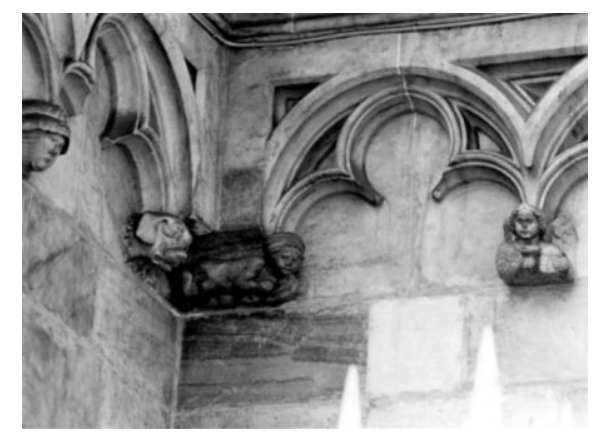

Fig. 4. Milano, Duomo: peducci della cornice esterna.

E' evidente che questo poteva essere reso possibile a partire da un comune substrato di esperienze artistiche, da una solida tradizione operativa e da un'effettiva peculiarità concettuale e pratica. Non escludo che uno dei motivi dei frequenti licenziamenti di architetti stranieri fossero proprio tali diversità, oltre a un certo corporativismo locale, come dimostrano i dibattiti del 1400 e 1401 con Jean Mignot, ${ }^{14}$ nel contesto dei quali la Fabbrica afferma risolutamente la peculiarità del fare artistico rispetto alla pura scienza progettuale (scientia est unum et ars est aliud), l'inscindibile unità tra elaborazione progettuale e tradizione architettonica e decorativa (quae facta sunt, sunt facta per geometriam et per praticam), fino alla rivendicazione per il Duomo di "cose nuove". Laddove la novità è il rispetto di una tradizione che permette di concepire il progetto come una continua elaborazione creativa, generata dal-

13 Annali, I, p. 19.

14 Annali, I, pp. 202-210, 224-229. Cfr. J.S. Ackermann. Ars sine scientia nibil est. Gothic Theory of Architecture at the Cathedral of Milan, in The Art Bulletin, XXXI, 1949, pp. 84-111. 
l'esperienza di cantiere, dai continui dibattiti e dalla ricerca della qualità formale non a priori, ma "decorando" progressivamente l'architettura, dalla sua genesi alla sua realizzazione materiale.

La figura più significativa di tale posizione, capace anche di uno slancio creativo assolutamente personale, è quella di Giovannino de Grassi, che nel 1389 esordisce nella fabbrica come pittore e nel 1391 viene nominato architetto, carica che ricoprirà fino alla morte nel 1398. ${ }^{15}$ La sua poliedrica attività, che lo vede al tempo stesso protagonista della miniatura viscontea, pittore, scultore e operoso anche in altre tecniche artistiche, è fondata su una singolare capacità disegnativa. La centralità del disegno nel lavoro di Giovannino trova conferma nelle continue forniture di carta da parte della fabbrica e nella gelosa protezione della stanza in cui opera ad designandum. Il disegno permette al maestro una continua elaborazione creativa, nel delicato passaggio tra rappresentazione naturale, ispirata alla molteplicità del reale, e raffinate soluzioni formali, valide per diverse applicazioni, ma in certi casi anche svincolate da esse e proiettate verso infiniti orizzonti fantastici, come in alcune pagine dell'Offiziolo Visconti. ${ }^{16}$

Giovannino disegna sezioni e alzati, ma anche piloni e capitelli, finestroni e strafori, sagome per colonnine e sculture, oreficerie e vetrate. Non si deve semplicemente pensare alla tradizione dei modelli medievali, tipo Villard de Honnecourt, quanto a una nuova sorgente generativa di forme: una nuova capacità d'indagare la natura e lo spazio e di esprimerli in un'infinita varietà creativa e formale, sorretta da uno straordinario controllo stilistico, capace di accogliere suggerimenti diversi e trascorrere da affilate e nervose eleganze a morbide e addolcite cadenze.

Un esito significativo dell'attività di Giovannino è il nuovo equilibrio formale che presenta la cattedrale milanese, ${ }^{17}$ nella quale la tensione tardogotica si placa in un più misurato senso spaziale - connotato dall'alzato ad triangulum stabilito nella riunione del primo maggio 1392

15 M. Rossi, Giovannino de Grassi, cit.; Id., Grassi, Giovannino, in Dizionario Biografico degli Italiani, LVIII, Roma 2002, pp. 640-647, anche per i successivi riferimenti documentari.

16 M. Rossi, Fantasie architettoniche di Giovannino de Grassi, in Arte Lombarda, 146/147/148, 2006, 1-3, pp. 45-54.

17 Tale intuizione, accompagnata da una precisa lettura della qualità architettonica del Duomo, si deve ad A.M. Romanini. L'architettura gotica in Lombardia, Milano-Pavia 1964, pp. 391-392, ripresa in Il Duomo di Milano, Milano 1973, p. 176. 
- che non si esaurisce però nell'impianto architettonico, ma si arricchisce continuamente di soluzioni decorative.

Ad esempio, nella nuova modulazione dello spazio tardogotico del Duomo, alla quale non è estranea la concezione "a sala" della tradizione gotica lombarda, assumono notevole importanza i grandi capitelli a tabernacolo (Fig. 5), che accompagnano il fedele lungo la navata fino al tiburio, dove la sequenza dei santi converge nella glorificazione teologica dell'intero spazio ecclesiale, della chiesa terrena in prospettiva celeste. La soluzione formale risulta inedita nel contesto dell'architettura tardogotica europea, che privilegiava le gallerie di santi sulle facciate, come a Strasburgo: qualificando lo spazio interno del Duomo, al tempo stesso ne modula lo slancio ascensionale, pausando la salita dei piloni e generando l'apertura delle volte a crociera.

Tale soluzione viene ovviamente criticata dagli architetti stranieri come il Mignot per gli squilibrati rapporti dei capitelli con le basi dei piloni: la Fabbrica risponde, in chiave preumanistica, che le proporzioni corrispondono a quelle tra la testa e i piedi nell'uomo (1:4), anzi la grandezza dei capitelli risulta aumentata di due braccia (per cui il rapporto diventa 1:5) propter ad ornamentum pironum pro ponendo figuras. ${ }^{18}$

La consapevolezza da parte della Fabbrica del valore decorativo e iconografico dei grandi capitelli a tabernacolo affonda le sue radici nel dibattito avviato fra il 1392 e il 1393, quando risulta in corso di esecuzione il primo capitello, che il Cadei ha giustamente individuato sul pilone 83, all'imbocco del coro (Fig. 5), attribuendolo a Giovannino de Grassi. ${ }^{19}$ Dopo diverse discussioni, il 17 aprile 1396 viene definitivamente approvata la forma elaborata da Giovannino ad tabernaculos, cum intentione intus ponendi figuras lapidum marmoreorum cum foliaminibus et pileis et strafuriis, con la possibilità di alcune varianti. ${ }^{20}$

Risulta affascinante, per l'accurata modulazione del ritmo ascensionale, come il capitello di Giovannino assecondi le membrature del pilone, favorendo, attraverso le nicchie per le statue, l'acuto insinuarsi delle ghimberghe tra i costoloni che aprono la volta e al tempo stesso pausando la spinta verso l'alto.

18 Annali, I, pp. 203-204.

19 A. Cadei, I capitelli più antichi del Duomo di Milano, in Il Duomo di Milano..., cit., 1969, pp. 77-88.

20 Annali, I, p. 162. 


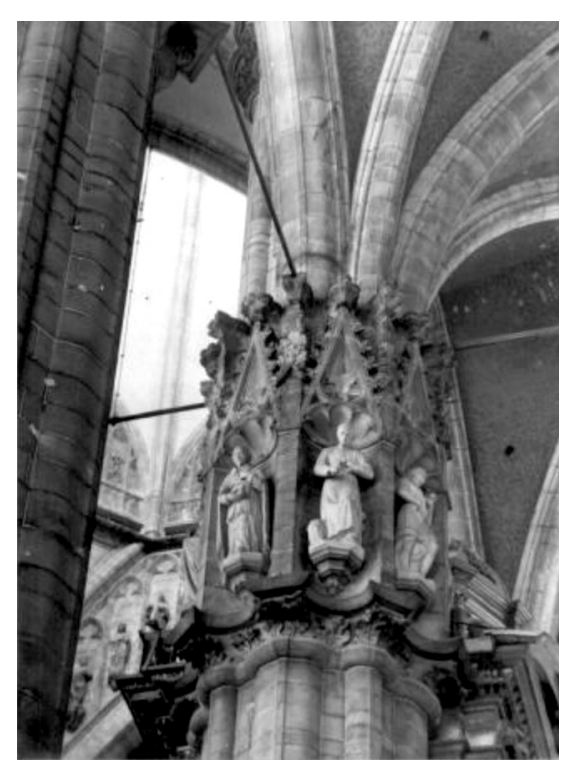

Fig. 5. Milano, Duomo. Giovannino de Grassi, capitello.

Il valore della qualità decorativa dell'architettura non si esaurisce comunque neppure in tale invenzione, ma, quasi in un intreccio tra sollecitazioni progettuali ed esuberanza creativa, si ritrova in alcune iniziali miniate del Beroldo, il manuale liturgico fatto miniare nel 1397/1398 a Giovannino e a suo figlio Salomone.

A tale contesto appartengono pure le riflessioni del maestro sul tiburio e le prime guglie del Duomo, svelate sul frontespizio dello stesso codice (Fig. 6), diverse e al tempo stesso aggiornate alle soluzioni che Ulrich von Einsingen andava proponendo per la cattedrale di Ulm. ${ }^{21}$ Rispetto all'indefinita moltiplicazione ascensionale delle strutture, Giovannino predilige una sintesi formale unitaria, intesa a indagare e al tempo stesso rappresentare i valori decorativi dell'architettura e dello spazio.

E'significativa pure la sintonia del disegno con alcune oreficerie, come il calice di Gian Galeazzo Visconti del Duomo di Monza, ${ }^{22}$ te-

21 Cfr. R. Wortmann, in Die Parler und der Schöne Stil 1350-1400. Europäische Kunst unter den Luxemburgern, I, Köln 1980, pp. 326-327; Les Bâtisseurs des Cathédrales gothiques, a cura di R. Recht, Strasbourg 1989, pp. 409-410.

22 L'intuizione si deve già a P. Toesca, La pittura e la miniatura nella Lombardia 
nendo conto del fatto che nel 1396 Giovannino lavora anche a un paliotto d'oro per l'altare maggiore del Duomo, in cui incastonare gioielli donati dalla duchessa. ${ }^{23}$

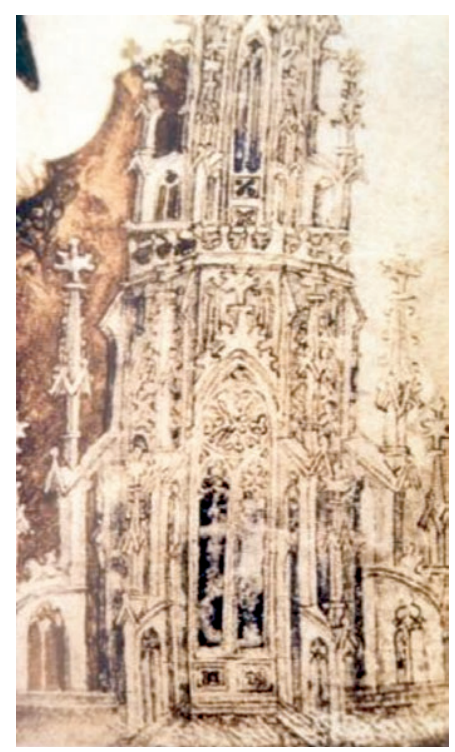

Fig. 6. Milano, Biblioteca Trivulziana, ms 2262, Beroldo, f. 1r. Giovannino e Salomone de Grassi, guglia, particolare.

Tornando alle guglie, l'unica costruita in questa prima fase del cantiere milanese è quella realizzata in seguito all'eredità lasciata dal banchiere Marco Carelli nel 1394 (Fig. 7), progettata probabilmente dallo stesso Giovannino. Ancora una volta il ritmo ascensionale è placato e modulato da una raffinata corona di ghimberghe, che si slanciano verso l'alto con caratteristici pinnacoli con gattonature a foglie chiuse e al

dai più antichi monumenti alla metà del Quattrocento, Milano 1912, p. 311. Per l'Ostensorio di Voghera (Milano, Musei Civici del Castello Sforzesco), spesso rapportato alle architetture di Giovannino (si veda ad esempio, con ampia riflessione critica, A. Cadei. Cultura artistica delle cattedrali: due esempi a Milano, in Arte Medievale, V, 1991/1, 83-103), è stata recentemente sostenuta la datazione al 1456 che compare sul piede (L. Cavazzini. Il crepuscolo della scultura medievale in Lombardia, Firenze 2004, 134-136).

23 Annali, I, pp. 163-164. 
tempo stesso sono scandite da una raffinata sequenza di statue di $A n$ geli musicanti, recentemente attribuiti dalla Cavazzini allo scultore francese Roland de Banille. ${ }^{24}$

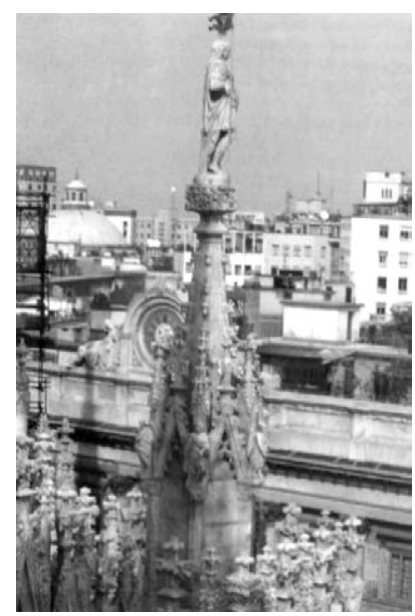

Fig. 7. Milano, Duomo: guglia Carelli.

Il naturalismo degli inserti figurativi, che ornano e assecondano plasticamente le strutture architettoniche del Duomo, insinuandosi elegantemente nelle volte e nelle guglie, nei portali e nei finestroni, ha avuto una sorprendente conferma in alcune immagini di statue dipinte ritrovate sulle piccole guglie della sovrapporta della sacrestia settentrionale (Fig. 8 e Fig. 9). ${ }^{25}$

L'impresa, alla quale lavorano Giovannino e il fratello Porrino nel 1395 , si colloca in un momento estremamente significativo della signoria viscontea, quando Gian Galeazzo ottiene la nomina a duca di Milano: vale la pena tener presente che proprio nelle sacrestie del Duomo furono celebrate particolari funzioni religiose durante i festeggiamenti seguiti all'incoronazione avvenuta nella basilica di S. Ambrogio. ${ }^{26}$

Le figure meglio conservate sono quelle di San Giorgio che uccide il drago e di San Cristoforo: la prima caratterizzata dal cavallo mirabil-

24 L. Cavazzini, Il crepuscolo della scultura..., cit., pp. 41-42.

25 M. Rossi, Novità su Giovannino de Grassi, in Arte Lombarda, 108/109, 1994, 1-2, pp. 51-55; Id., Fantasie architettoniche..., cit., p. 45.

26 Annali, App., I, p. 236. 
mente scorciato nel ristretto spazio del pilastrino, come accade nel ductus di alcune lettere figurate del Taccuino di Bergamo, e la seconda dal linearismo disegnativo morbidamente addolcito dal colore. Il pinnacolo coronato alla base da ghimberghe che sovrasta il S. Giorgio richiama la guglia Carelli, ma ancora una volta pure alcune soluzioni orafe del già citato calice del Duomo di Monza.

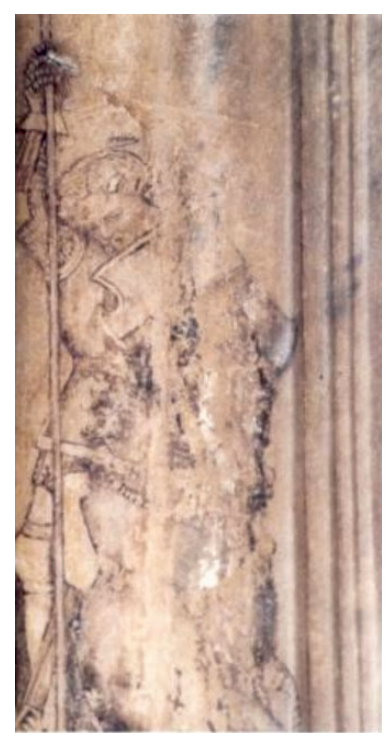

Fig. 8. Milano, Duomo, sovrapporta della sacrestia settentrionale.

Giovannino de Grassi, S. Giorgio uccide il drago, particolare.

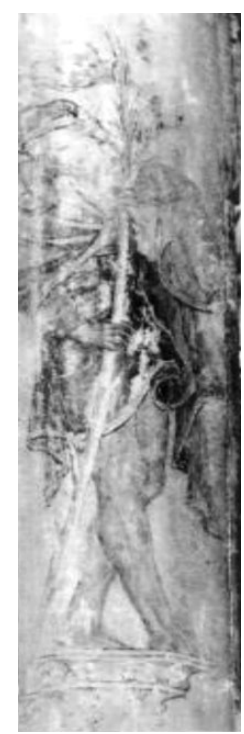

Fig. 9. Milano, Duomo, sovrapporta della sacrestia settentrionale.

Giovannino de Grassi,

S. Cristoforo.

La circolazione di disegni nell'ambito di differenti contesti e la loro adozione in opere realizzate con tecniche diverse è verificabile anche in alcune mensole del primo ordine di statue all'esterno del Duomo di Milano (Fig. 10), per le quali è documentato l'intervento pittorico di Giovannino: ${ }^{27}$ in particolare raffigurano Re biblici e Profeti, connotati da arditi scorci e ricchi inserti naturalistici e con caratteristici dettagli iconografici, come i cartigli e i libri sorretti dai personaggi rappresentati con incisi i loro nomi. Figure scorciate che reggono iscrizioni si tro-

27 Annali, App. I, p. 231. Cfr. M. Rossi. Giovannino de Grassi..., cit., pp. 107-109. 
vano pure nell'Offiziolo Visconti BR 397 (Firenze, Biblioteca Nazionale), ad esempio gli Angeli miniati a f. 122v.

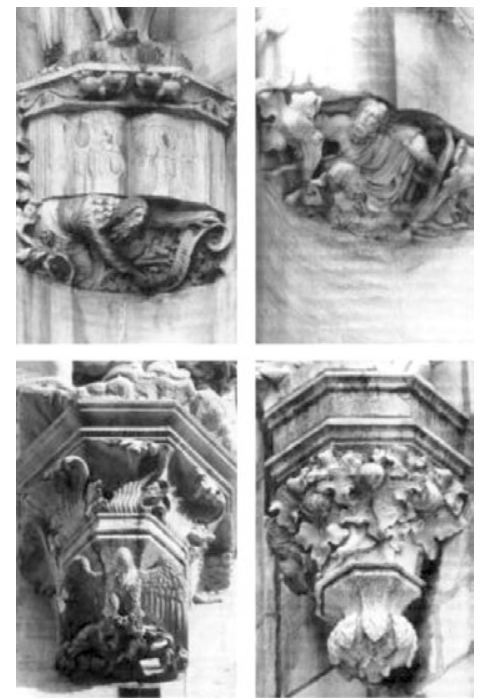

Fig. 10. Milano, Duomo: mensole del primo ordine esterno di statue.

Spesso gli interventi decorativi ispirati da disegni del maestro sono dunque caratterizzati, oltre che da evidenti stilemi, da invenzioni iconografiche e figurative degne di rilievo: insieme ai citati libri, segnaliamo i cartigli con i nomi dei santi avvolti intorno a singolari efflorescenze con radici che s'insinuano nelle strombature dei finestroni (Fig. 11), significative espressioni del gusto naturalistico di Giovannino, estremamente vitale. I cartigli attorcigliati a motivi fitomorfi si ritrovano pure in alcune iniziali miniate del Beroldo, come ad esempio a f. 241v, mentre nel margine destro della Creazione del cielo e della terra a f. 19r dell'Offiziolo Visconti LF 22 si avvolgono attorno a una guglia. Nelle pagine iniziali di questo codice, direttamente realizzate o ispirate dal maestro, ${ }^{28}$ le bordure miniate trascorrono liberamente da fantasiose guglie architettoniche a sottili tronchi d'alberi, come a f. 30v, in un continuo, mutevole scambio creativo tra natura e architettura.

28 Si veda, anche per la bibliografia precedente, M. Bollati. La miniatura tardogotica in Lombardia e il Libro d'ore Visconti, in Il Libro d'ore Visconti. Commentario al codice, Modena 2003, pp. 302 sgg. 


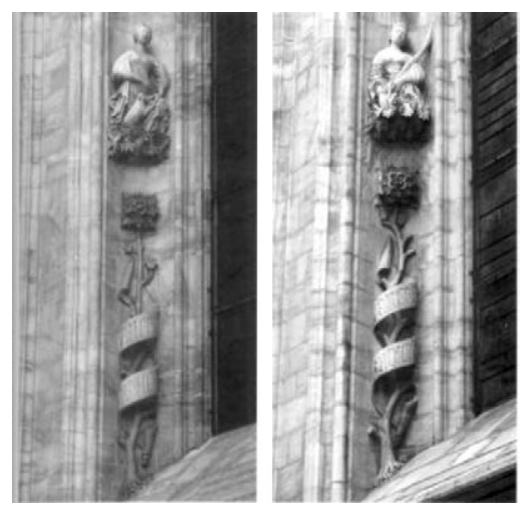

Fig. 11. Milano, Duomo: finestroni, particolari delle strombature.

I disegni di Giovannino, gelosamente custoditi dalla Fabbrica del Duomo, avranno fortuna anche dopo la sua morte, soprattutto per quanto riguarda i dettagli decorativi e le statue, come testimoniano alcuni Giganti con i relativi doccioni, quali l'Homo selvaticus, ispirato a quello disegnato nel Taccuino di Bergamo, l'Uomo d'arme con una mazza ferrata, ${ }^{29}$ che ricorda le lettere E e D dell'alfabeto figurato, e quello del transetto settentrionale con un grande nodo alla cintura, ${ }^{30}$ che richiama il fiocco a f. 19r del codice bergamasco.

Tra gli scultori attivi all'inizio del Quattrocento nell'impresa dei Giganti, che connotano il coronamento superiore della decorazione esterna del Duomo con motivi iconografici che trascorrono dalla tradizione biblica a quella classica, sono stati individuati importanti scultori come Alberto da Campione e Jacopino da Tradate. Il primo, messo recentemente in luce dalla Cavazzini nei cantieri delle cattedrali di Milano, Como e Bologna, scolpisce statue "di infuocata temperatura espressiva", tra le quali la chiave di volta del deambulatorio con il profeta Abdia e due possenti Giganti dell'abside nel 1403 (Fig. 12).31

All'impresa dei Giganti, oltre a Matteo Raverti, Giorgio Solari e altri scultori, lavora anche Jacopino da Tradate, ${ }^{32}$ che sarà celebrato nell'iscrizione posta sotto la statua di Martino V: in arte profundus nec Prasitele minor sed maior.

29 U. Nebbia, La scultura nel Duomo di Milano, Milano 1908, n. 54.

30 U. Nebbia, La scultura...., cit., n. 82.

31 L. Cavazzini, Il crepuscolo della scultura..., cit., pp. 17 sgg.

32 L. Cavazzini, Il crepuscolo della scultura..., cit., pp. 55 sgg. 


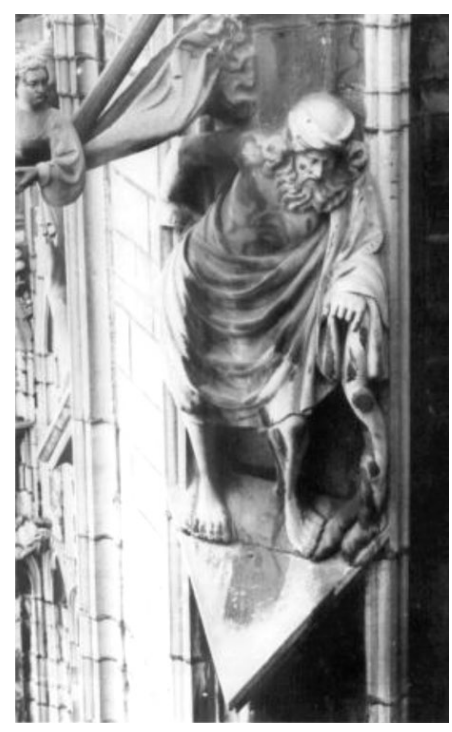

Fig. 12. Milano, Duomo. Alberto da Campione, Gigante.

Lo scultore esordisce nel cantiere del Duomo nel 1401, realizzando un Angelo per un capitello di un pilone del coro; sei anni dopo è già a capo dei lapicidi e intorno al 1420 scolpisce il grandioso monumento di Martino V (Fig. 13), in ricordo della consacrazione dell'altare maggiore (1418), operata dal papa di ritorno a Roma dal Concilio di Costanza. Jacopino traduce il decorativismo tardogotico e gli stilemi internazionali sui quali si era formato, oltre che su Bonino da Campione, in un nuovo linguaggio, equilibrato e al tempo stesso sontuoso, morbidamente atteggiato e raffinatamente perseguito, che potremmo qualificare come l'approccio milanese alla cultura umanistica.

Negli stessi anni, e oltre, è documentato in Duomo un altro grande artista, Michelino da Besozzo, celebrato dalla Fabbrica summus in arte pictoria et designamenti, ex quo speratur quam multus erit utilis, ${ }^{33}$ il più diretto parallelo di Jacopino. Dopo il soggiorno in Veneto, nel 1418 decora la Madonna nella chiave di volta del coro attri-

33 Annali, I, p. 261. Cfr. M. Rossi. Una Maestà di Michelino da Besozzo per Santa Tecla, in Arte e storia di Lombardia. Scritti in memoria di Grazioso Sironi, Roma 2006 (Biblioteca della Nuova Rivista Storica, n. 40), pp. 31-36. 
buita proprio a Jacopino, ${ }^{34}$ e nel 1421 dipinge l'altare dei SS. Quirico e Giulitta, per il quale realizza anche le vetrate in diversi intervalli di tempo (Fig. 14).

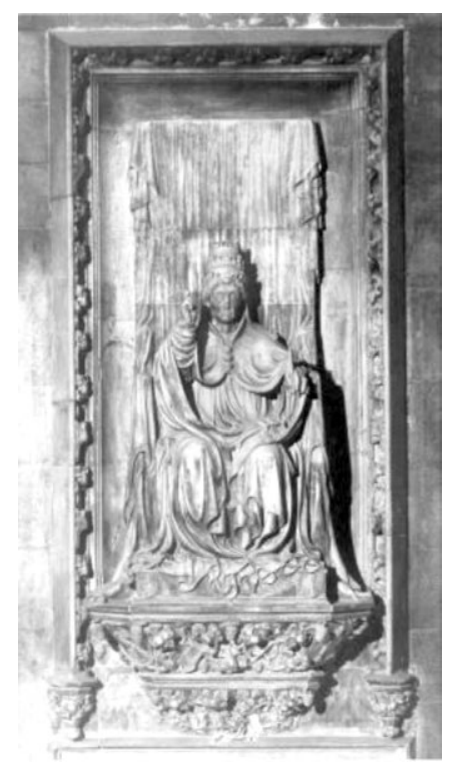

Fig. 13. Milano, Duomo. Jacopino da Tradate, Monumento a papa Martino V.

Come Giovannino de Grassi, anche Michelino testimonia un'intensa attività di raccordo tra le arti, favorita dal disegno quale principio generativo della produzione figurativa. I suoi disegni per sculture e vetrate, così come i dipinti e le miniature costituiscono un imprescindibile punto di riferimento per la cultura artistica milanese, in una suggestiva e inesauribile tensione espressiva fra le delicate modulazioni di un sogno pressoché irraggiungibile e la coscienza di una profondità umana che traspare dai volti (Fig. 14).

Diversamente da Giovannino, né Jacopino né Michelino sembrano occuparsi di architettura, forse per una certa stasi del cantiere, forse perché per le opere in corso erano già sufficienti i disegni esistenti e la tradizione operativa delle maestranze, forse anche per una progressiva specializzazione delle stesse.

34 Annali, App., I, p. 319; L. Cavazzini. Il crepuscolo della scultura ..., cit., p. 83. 


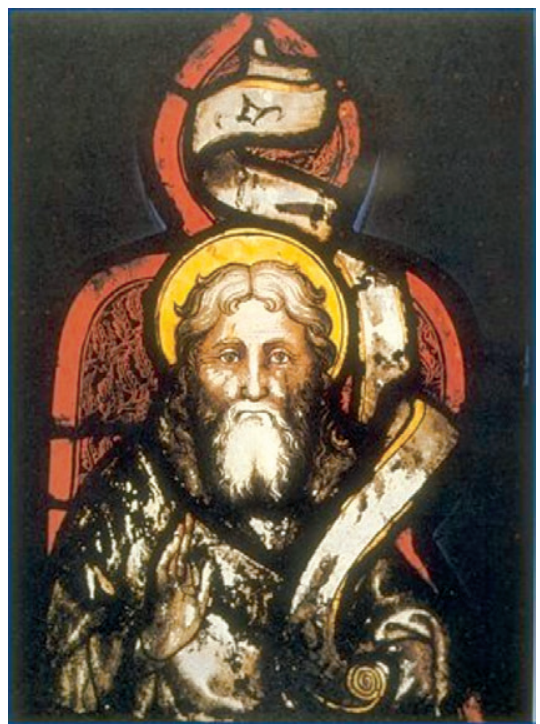

Fig. 14. Milano, Duomo. Michelino da Besozzo, vetrata di S. Giulitta, particolare.

E' impressionante in questi anni la densità d'interventi pittorici e di lavori alle vetrate: $i$ dati documentari a disposizione contribuiscono a offrirci un panorama peculiare dell'organizzazione artistica a Milano, in quanto il cantiere della cattedrale tardogotica offriva numerose possibilità di lavoro ai pittori, forse più che la tradizionale attività delle botteghe. 

